\title{
Peripheral vascular stiffness, assessed with two-dimensional speckle tracking versus the degree of coronary artery calcification, evaluated by tomographic coronary artery calcification index
}

\author{
Michał Podgórski¹, Piotr Grzelak , Konrad Szymczyk ${ }^{1}$, Ewa Szymczyk², Jarosław Drożdż², \\ Ludomir Stefańczyk ${ }^{1}$
}

1Department of Radiology and Diagnostic Imaging, Medical University of Lodz, Lodz, Poland

${ }^{2}$ Department of Cardiology, Medical University of Lodz, Lodz, Poland

Submitted: 10 October 2012

Accepted: 20 December 2012

Arch Med Sci 2015; 11, 1: 122-129

DOI: 10.5114 /aoms.2015.49205

Copyright @ 2015 Termedia \& Banach

\section{Abstract}

Introduction: Even in asymptomatic patients, the result of atherosclerosis progression is deterioration of the function and morphology of the artery wall. Two-dimensional speckle-tracking (2DST) is a sonographic technique that allows for precise evaluation of arterial wall compliance. Together with measurement of intima-media thickness (IMT), it can be applied for quick and non-invasive assessment of the progression of peripheral artery atherosclerosis.

Material and methods: Fifty-eight patients of mean age 61 years (SD 10.6) underwent cardiac computed tomography (CT) and subsequent ultrasonographic evaluation of the left common carotid artery. The calcium score was calculated according to the Agatston method and compared with IMT, circumferential strain variables assessed by 2DST, conventional arterial stiffness parameters ( $\beta$-stiffness index and elastic modulus) and clinical data. Intra-observer and inter-observer agreement was evaluated.

Results: Strain variables and IMT differed significantly in patients with calcium score $(\mathrm{CS})>0$ and $\mathrm{CS}=0$. Moreover, they correlated with $\mathrm{CS}$, systolic blood pressure and age of patients. Conventional stiffness parameters were not able to identify the group of patients with calcifications present in the coronary arteries. For the 2DST technique, interclass and intraclass agreements were $84.83 \%$ and $94.42 \%$ respectively.

Conclusions: Circumferential strain variables assessed by 2DST and measurement of IMT can be used for evaluation of peripheral artery deterioration in patients until the $6^{\text {th }}$ decade of life. These parameters reflect the development of calcifications in coronary arteries and, more importantly, can be used for a more detailed estimation of the atherosclerosis risk in patients with $\mathrm{CS}=0$.

Key words: circumferential strain, circumferential strain rate, common carotid artery ultrasonography, intima-media thickness, cardiac computed tomography.

\section{Introduction}

Arteriosclerosis is a global process of arterial wall hardening. Significant alterations of the mechanical properties of the vessel walls may oc-

\author{
Corresponding author: \\ Piotr Grzelak MD, PhD \\ Department of Radiology \\ and Diagnostic Imaging \\ Medical University of Lodz \\ 22 Kopcinskiego St \\ 90-159 Lodz, Poland \\ Phone: +48 426786734 \\ Fax: +48 426781176 \\ E-mail: \\ piotr.grzelak@umed.lodz.pl
}


cur before any anatomical changes become visible $[1,2]$. Recognising patients with increased arterial stiffness is vital because they are at risk of myocardial infarction, stroke, coronary artery disease and hypertension [3-5]. As the reduced vessel wall compliance may be not just a surrogate of the arteriosclerosis but also its precursor, proper and early treatment should be introduced even in very young individuals $[4,6]$. There is no consensus as to which technique should be used and which vessel should be examined for the most reliable evaluation of arterial tree stiffening [4, 7-11]. Recently the two-dimensional speckle-tracking (2DST) technique, which was primarily a cardiac application [12], has enabled the estimation of arterial wall motion and deformation (strain) during the cardiac cycle $[3,13]$. Nevertheless, for the direct assessment of heart artery calcification, the tomographic coronary artery calcification index (calcium score - CS) should be calculated [14]. Unfortunately, cardiac computed tomography (CT) not only is associated with high doses of radiation but also requires patients to have a stable heart rate. Hence, the aim of this study was to evaluate the correlation of CS values with carotid artery function and morphology as assessed by 2DST/ IMT. It is hypothesised that sonographic evaluation of common carotid artery (CCA) wall deterioration will allow the identification of patients with calcifications in coronary arteries.

\section{Material and methods}

The analysed population consisted of 80 patients who were referred for cardiac tomography examination due to cardiac disorders. Of the initial group, the calcium score could not be calculated in 22 patients due to earlier coronary artery stent placement, presence of bypasses or atrial fibrilla- tion. The medical history was derived from medical records and from the interview. The following cardiovascular risk factors were evaluated: hypertension (blood pressure $>140 / 90 \mathrm{~mm} \mathrm{Hg}$ on repeated measurements or taking an antihypertensive medication), diabetes (fasting plasma glucose level $>126 \mathrm{mg} / \mathrm{dl}$, taking hypoglycaemic drugs or insulin, or a combination of these), hypercholesterolaemia (total cholesterol level > $220 \mathrm{mg} / \mathrm{dl}$ or receiving strain treatment) and smoking history.

\section{Cardiac computed tomography and calcium score calculation}

Multi-slice CT scanning of the thorax was performed with a GE Light-Speed scanner to determine the degree of coronary artery calcification (CAC). The acquisition was performed using prospective ECG triggering to eliminate motion artefacts, with a $50 \mathrm{~cm}$ scan field of view. The images were reconstructed with a standard algorithm using a $512 \times 512$ matrix. Voxels with a density equal to or higher than 130 Hounsfield units (HU) were recognised as calcifications (Figure 1). These were counted and the results were analysed using CardIQ Smart Score software (General Electrics). The calcification score was determined using the Agatston scoring system, with a score above zero considered as positive for the presence of calcium.

\section{Carotid ultrasonography}

After a rest of at least 5 min in a semi-dark, quiet room, the blood pressure in the left upper limb was measured with an semi-automatic sphygmomanometer. Once an electrocardiogram trace was obtained, the patients were examined in a supine position with the head turned $45^{\circ}$ to the right side. Examinations were performed using a Vivid 7 ap-
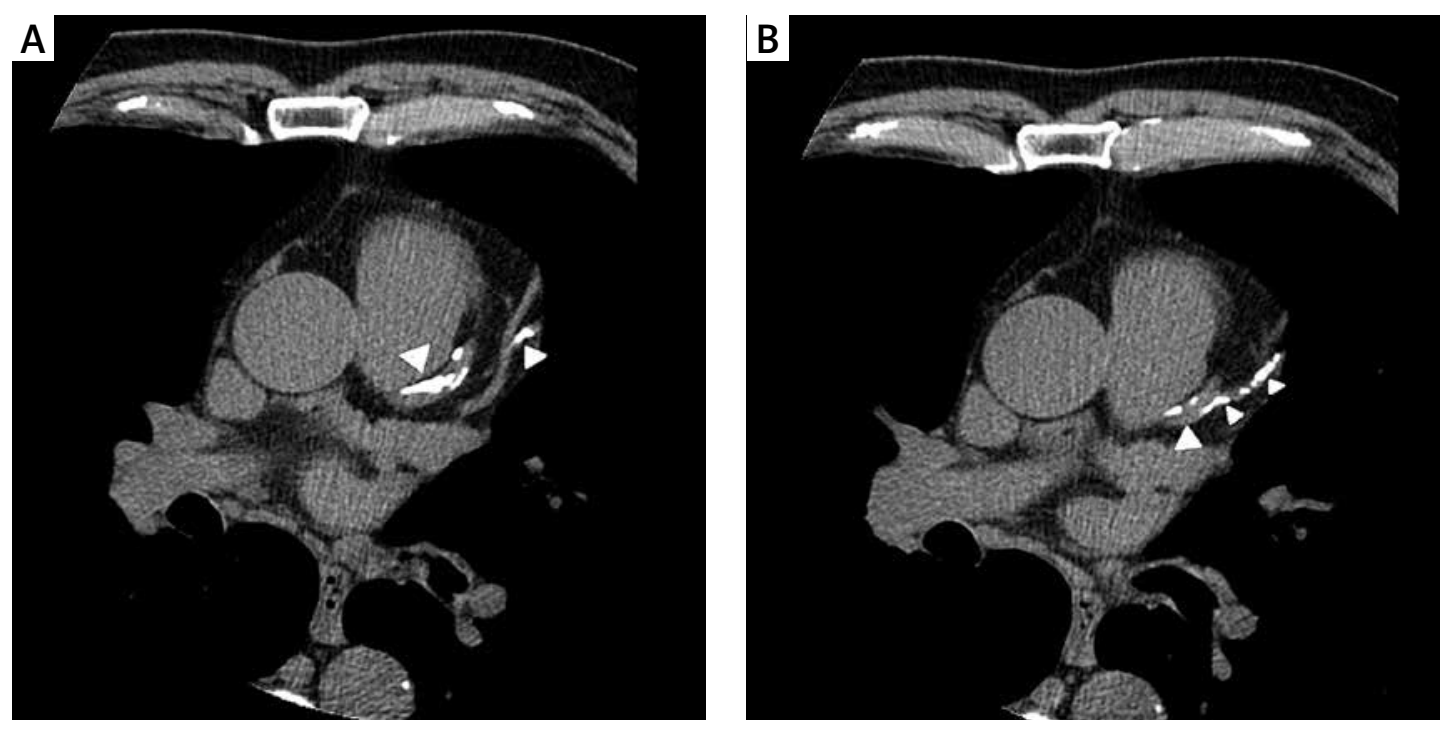

Figure 1. Multi-slice heart CT with calcifications in the left anterior descending artery and its branches (arrowheads) 


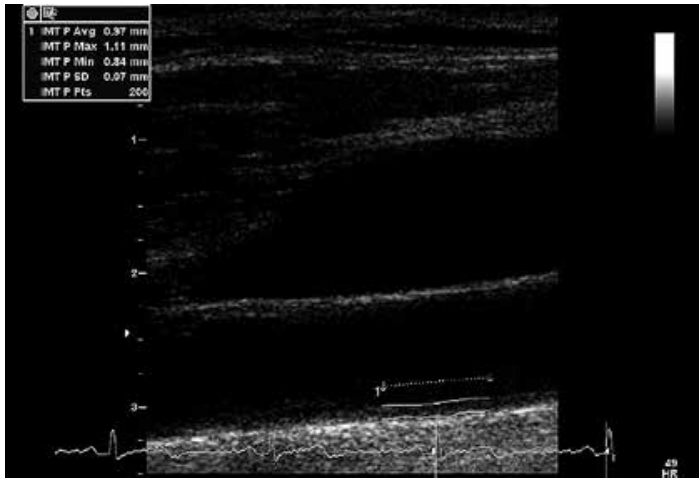

Figure 2. Measurement of the IMT on the far wall of the CCA

paratus (GE Vingmed Ultrasound) with a $14 \mathrm{MHz}$ linear transducer.

Long axis images of the left CCA were obtained up to the carotid bulb. The intima-media complex was visualised and cine loops of three cardiac cycles were saved. Afterwards, cine loops of three cardiac cycles of the short axis were acquired in an arterial segment about $1 \mathrm{~cm}$ below the carotid bulb. No atherosclerotic plaque was found in the analysed segment in any patient. To minimize the motion artefacts, all acquisitions were performed during a short breath-hold at the end of expiration. To allow stain assessment in all examinations, the frame rate was at least 70 frames per second. Further analyses were performed off-line on a workstation equipped with dedicated software (EchoPac PC, GE Medical System). Mean IMT for the far wall was measured at the $\mathrm{R}$ wave on the electrocardiogram. Evaluation was performed by means of a semi-automated border-detection program at a distance of 200 points proximal from the carotid bulb (Figure 2).

The $\beta$-stiffness index and elastic modulus (Ep) were assessed as conventional parameters characterising arterial stiffness. For their calculation, long-axis cine loops were used. M-mode analysis was carried out in the region $1 \mathrm{~cm}$ below the carotid bulb. The distance between the lumen-intima echo border of the near and the far walls was measured in 3 consecutive heart beats and averaged. Elasticity variables were calculated according to the following formulae:

$\beta=\frac{\ln (P s / P d)}{(D s-D d) \mid D d}, E p=\frac{(P s-P d)}{(D s-D d) \mid D d}$,

where $D d$ and $D s$ were, respectively, the diastolic and systolic lumen diameters, and $P S$ and $P d$ were the respective systolic and diastolic blood pressures. The elastic modulus was presented in kilopascals $(\mathrm{kPa})$.

For measurements of circumferential strain and strain rate, the lumen-intima echo border was circumscribed and the region of interest (ROI) was automatically marked externally to the line. To cover the cross-sectional area of the CCA wall, the ROI width was adjusted. Adequate tracking for all six segments was verified and, if necessary, the ROI was recalculated. 'Global' values for the whole ROI were assessed. Minimal and maximal circumferential strain (\%) as well as strain rate (strain per time unit, 1/s) were measured for 3 heart beats of the cine loop (Figure 3). The amplitudes were calculated and averaged. For the calculation of interclass and intraclass agreement, an additional 20 off-line analyses were performed by 2 blinded observers and by the same observer at 2 different time points. Only the peak circumferential strain was evaluated for one cardiac cycle automatically selected by the software.

\section{Statistical analysis}

Continuous variables are expressed as the mean \pm standard deviation (SD) and categorical variables
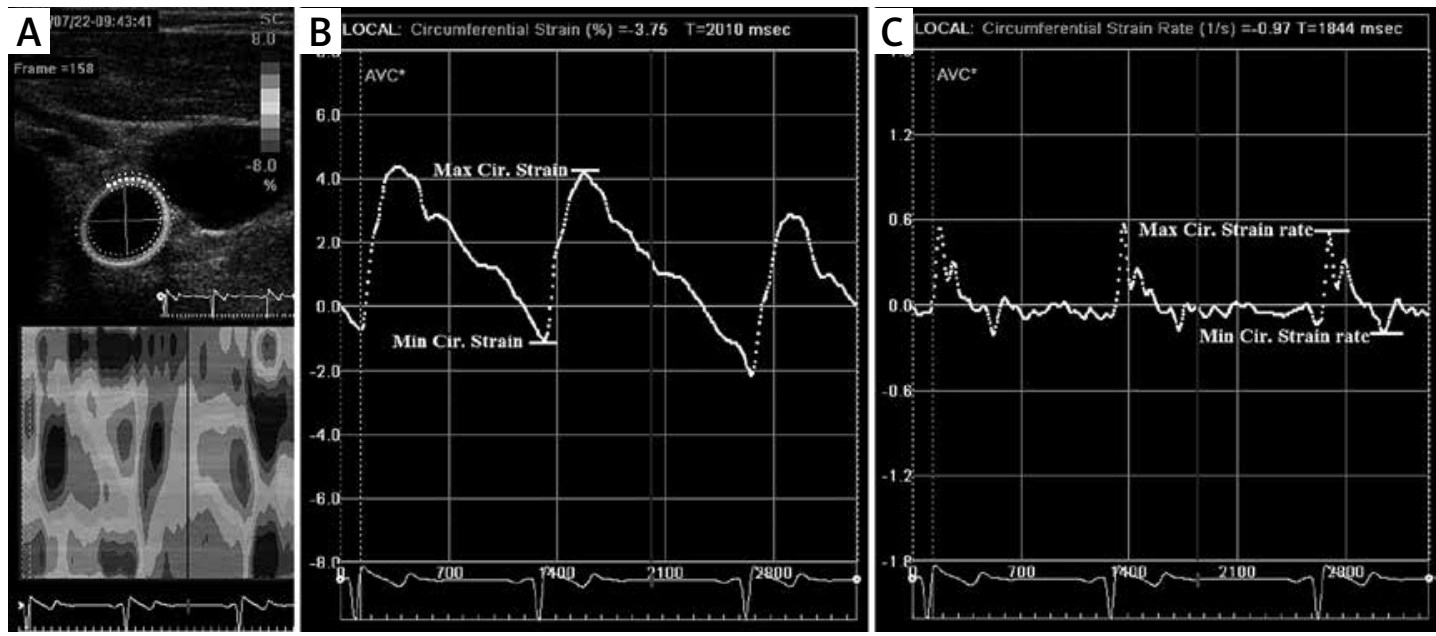

Figure 3. Evaluation of strain variables. Circumscribed ROI in CCA (A). Plots of the global circumferential strain (B) and circumferential strain rate (C) for three consecutive heart beats with marked maximal (Max) and minimal (Min) values of strain variables 
as frequencies and percentages. The Shapiro-Wilk test was used to determine the normality of the data. A Box-Cox transformation was performed for the parameters with skewed distribution. Differences in strain variables, arterial elasticity indices, mean IMT and clinical data between patients with $\mathrm{CS}=0$ and $\mathrm{CS}>0$ were tested with the $t$-tests for independent samples, Mann-Whitney test and by means of the $\chi^{2}$-squared test: Yates-corrected in the case of group sizes below 15 or two-tailed Fisher's exact tests for groups smaller than five. The correlation between continuous data was evaluated by Spearman's correlation coefficient. The results of interclass and intraclass agreement were analysed with the Bland-Altman plot and by calculating the $R^{2}$ value by means of linear regression analysis. The Bland-Altman plot depicts the percentile difference between two measurements ( $y$-axis) against their mean ( $x$-axis) and is used to assess for bias. The $R^{2}$ value gives the level of agreement. Statistical analysis was performed using Statistica for Windows (version 10.0, StatSoft, Tulsa, OK, USA). A $p$-value of $\leq 0.05$ was considered significant.

\section{Results}

The analysed population comprised 58 individuals (35 females; $60.34 \%$ ) with a mean age of 61 years
$(S D=10.6)$. The characteristics of the groups with $\mathrm{CS}=0$ and $\mathrm{CS}>0$ are presented in Table I. Patients with the presence of calcium in coronary arteries $(\mathrm{CS}>0)$ had significantly decreased strain variables and increased IMT (Figure 4).

The total amount of calcium deposits correlated significantly with the circumferential strain (Figure 5) and strain rate, as well as with IMT. The circumferential strain rate and IMT also correlated with each other. Correlations of circumferential strain and IMT with age and systolic blood pressure (SBP) were also significant (Table II). The $\beta$-stiffness index and elastic modulus correlated neither with the CS nor with IMT and strain variables. Interclass and intraclass agreement were $84.83 \%$ and $94.42 \%$ respectively. Information about the bias and limits of agreement between observers is presented in the Bland-Altman plot (Figure 6).

\section{Discussion}

There is a growing need for a simple, reliable and non-invasive method of detecting early disturbances in arterial wall morphology and function [15]. Decreased vessel compliance is associated with several cardiovascular risk factors [16], but only recently it has been defined as an inde-

Table I. Characteristic of patients with CS $=0$ and CS >0

\begin{tabular}{|c|c|c|c|}
\hline Parameter & $\begin{array}{l}\text { Calcium score }=0 \\
\qquad(N=30)\end{array}$ & $\begin{array}{l}\text { Calcium score }>0 \\
\quad(N=28), n(\%)\end{array}$ & Value of $p$ \\
\hline \multicolumn{4}{|l|}{ Gender, $n(\%)$} \\
\hline Female & $21(36.21)$ & $13(22.41)$ & \multirow[t]{2}{*}{0.12004} \\
\hline Male & $9(15.52)$ & $15(25.86)$ & \\
\hline Age, mean \pm SD & $56.8 \pm 10.1$ & $66.2 \pm 9.3$ & $0.000536^{*}$ \\
\hline Hypertension, $n$ (\%) & $16(27.59)$ & $19(32.76)$ & 0.38907 \\
\hline Diabetes, $n(\%)$ & $6(10.34)$ & $4(6.9)$ & 0.73159 \\
\hline Hypercholesterolaemia, $n(\%)$ & $10(17.24)$ & $13(22.41)$ & 0.45315 \\
\hline Cigarette pack years, mean \pm SD & $9.33 \pm 11.5$ & $16.79 \pm 17.4$ & 0.114246 \\
\hline $\begin{array}{l}\text { Mean blood pressure } \\
{[\mathrm{MBP}=\mathrm{DBP}+1 / 3(\mathrm{SBP}-\mathrm{DBP})] \text {, mean } \pm \mathrm{SD}}\end{array}$ & $12.48 \pm 1.5$ & $13.36 \pm 1.95$ & 0.059722 \\
\hline Systolic blood pressure, mean $\pm \mathrm{SD}[\mathrm{kPa}]$ & $16.82 \pm 2.17$ & $18.63 \pm 2.77$ & $0.007304^{\star}$ \\
\hline \multicolumn{4}{|l|}{ Artery diameter, mean \pm SD: } \\
\hline Systolic & $0.64 \pm 0.12$ & $0.71 \pm 0.13$ & 0.053113 \\
\hline Diastolic & $0.59 \pm 0.11$ & $0.65 \pm 0.13$ & 0.074300 \\
\hline \multicolumn{4}{|l|}{ Arterial elasticity indices, mean \pm SD: } \\
\hline$\beta$ stiffness & $7.74 \pm 5.09$ & $8.74 \pm 5.15$ & 0.475914 \\
\hline Elastic modulus [kPa] & $107.5 \pm 72.66$ & $129 \pm 79.41$ & 0.983092 \\
\hline Mean intima-media thickness, mean \pm SD & $0.53 \pm 0.15$ & $0.64 \pm 0.19$ & $0.008307^{*}$ \\
\hline \multicolumn{4}{|c|}{ Circumferential strain variables amplitudes, mean \pm SD: } \\
\hline Strain (\%) & $4.11 \pm 1.53$ & $3.23 \pm 1.36$ & $0.001954^{*}$ \\
\hline Strain rate $(1 / s)$ & $0.53 \pm 0.21$ & $0.4 \pm 0.15$ & $0.010699^{\star}$ \\
\hline
\end{tabular}



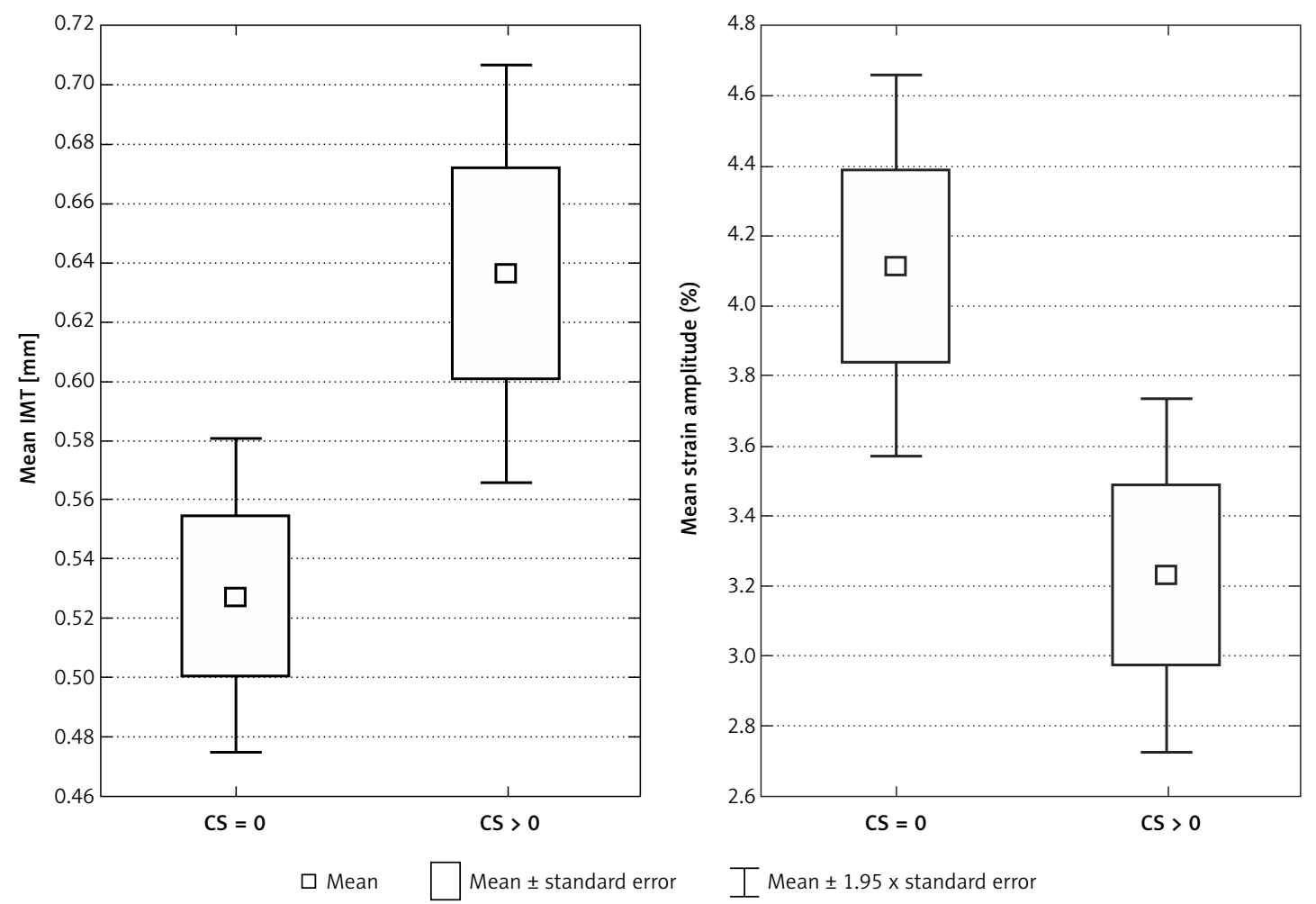

Figure 4. Difference of mean IMT and mean circumferential strain amplitude between groups with CS $=0$ and CS $>0$

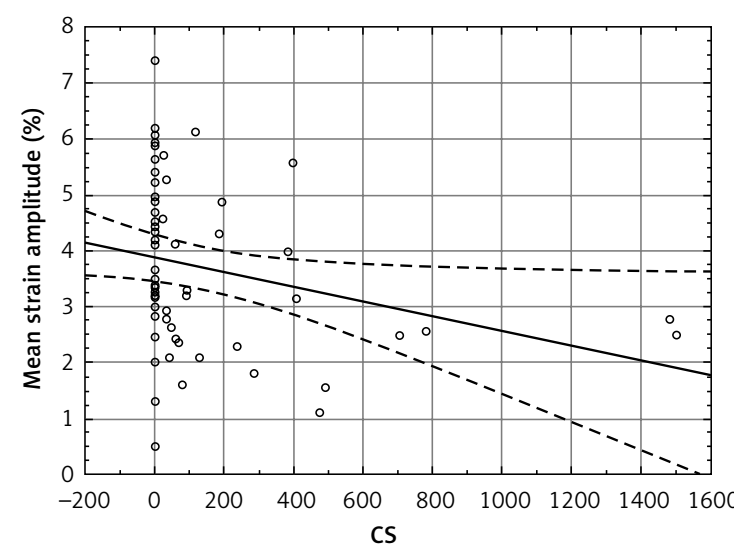

Figure 5. Correlation of CS values with mean circumferential strain amplitude

pendent predictor of all-cause and cardiovascular mortality, coronary events and stroke [17].

Assessment of arterial wall hardening is challenging due to its multifactorial nature. Non-homogeneous arterial wall structure [18, 19], actual smooth muscle tone [1] and measurement of the blood pressure in the brachial artery instead of the arterial site of interest can result in unreliable evaluation of stiffness indices [20]. In addition, although the elastic modulus is independent of arterial geometry, it is pressure dependant. The $\beta$-stiffness index is calculated based on a one-dimensional approach, giving it various other limitations [21]. Hence, there are many possible reasons why, unlike 2DST/IMT, these analysed parameters are not able to differentiate groups of patients with CS $=0$ and those with CS $>0$. It is also consistent with other studies which have proved the superiority of 2DST over Ep and $\beta$-stiffness index measurements as a way of detecting patients with hypertension [21], as well as over the evaluation of age-dependent differences in the elastic properties of the CCA [13]. On the other hand, Oishi et al. [19], who applied the 2DST in the evaluation of age-related changes in the abdominal aorta of 39 patients, recommend the use of strain in younger persons, and stiffness parameters in older individuals. This conclusion might be correct for the aorta which has stiffened more than the carotid artery [8], but in the present research concerning the CCA, the strain variables were found to be more suitable than conventional stiffness indices. Moreover, in routine clinical practice, the imaging of the CCA is much more convenient and has a number of advantages over aorta wall evaluation [13].

Two-dimensional speckle-tracking of the CCA is a highly feasible method for assessing subclinical atherosclerosis, and it improves the identification of patients with a higher risk of atherosclerosis [22]. It was also superior to IMT measurement in predicting coronary artery disease [10]. Furthermore, in patients with a good distribution of common cardiovascular risk factors, this method allows for better risk stratification compared with the assessment of the cardiovascular risk score alone [3]. 
Table II. Correlations between assessed parameters

\begin{tabular}{|c|c|c|c|c|}
\hline Parameter & CS & $\begin{array}{l}\text { Strain } \\
\text { circumferential } \\
\text { rate }(1 / s)\end{array}$ & $\begin{array}{c}\text { Mean } \\
\text { circumferential strain } \\
\text { amplitude (\%) }\end{array}$ & Mean IMT [mm] \\
\hline Age & $0.47\left(p=0.0002^{*}\right)$ & $-0.49\left(p<0.001^{\star}\right)$ & $-0.38\left(p=0.003^{\star}\right)$ & $0.41\left(p=0.0011^{\star}\right)$ \\
\hline$\beta$-Stiffness & $0.10(p=0.4515)$ & $-0.12(p=0.213)$ & $-0.01(p=0.9516)$ & $-0.06(p=0.6643)$ \\
\hline Elastic modulus [kPa] & $0.15(p=0.2601)$ & $-0.16(p=0.2438)$ & $-0.04(p=0.7524)$ & $0.01(p=0.9118)$ \\
\hline Calcium score & & $-0.4\left(p=0.002^{*}\right)$ & $-0.39\left(p=0.0027^{\star}\right)$ & $0.28\left(p=0.0328^{\star}\right)$ \\
\hline Systolic blood pressure [kPa] & & $-0.1283(p=0.337)$ & $-0.3280\left(p=0.012^{\star}\right)$ & $0.33\left(p=0.0109^{\star}\right)$ \\
\hline Mean blood pressure $[\mathrm{kPa}]$ & & $-0.1827(p=0.170)$ & $-0.2878\left(p=0.028^{\star}\right)$ & $0.33\left(p=0.0127^{\star}\right)$ \\
\hline $\begin{array}{l}\text { Circumferential strain rate } \\
(1 / \mathrm{s})\end{array}$ & & & & $-0.32\left(p=0.0154^{\star}\right)$ \\
\hline $\begin{array}{l}\text { Mean circumferential strain } \\
\text { amplitude (\%) }\end{array}$ & & & & $-0.24(p=0.0699)$ \\
\hline
\end{tabular}

However, there is no consensus about the CS cut-off point that would significantly increase the risk of cardiovascular events. It is well known that the CS cannot be used for direct quantification of the severity of coronary artery stenosis. Moreover, it is only in the absence of any calcifications $(C S=0)$ that advanced luminal disease can be excluded [23]. Despite this, in the present study, patients with $\mathrm{CS}=0$ formed a very heterogeneous group regarding strain variables and IMT. They were also significantly younger. This suggests that although increased arterial stiffness is usually accompanied by coronary artery calcifications in elderly patients, changes in arterial wall function and morphology precede the development of calcifications in the arteries of the heart in younger individuals. Hence, ultrasonographic parameters seem to better reflect the advancement of atherosclerosis in patients without calcifications in coronary arteries, and can supply additional information that would help to differentiate patients who have $\mathrm{CS}=0$ but might be at risk of faster atherosclerosis progression.

Increased IMT has been validated as a surrogate marker of cardiovascular events [3], cardiovascular disease [24, 25] and atherosclerosis [26]. Association of the IMT with CS has already been described [27], but the correlation of IMT with circumferential strain was found to be significant only in some studies [3, 10, 22, 28]. In others, including the present study, the correlation was not significant $[8,29]$. This suggests that changes in IMT and arterial stiffness may represent different aspects of atherosclerosis $[3,5]$. It is stated that structural abnormalities start reflecting atherosclerosis severity beyond a certain level [8] and that the arterial stiffness cannot increase beyond a certain maximal limit, which would restrict the predictive value of strain variables for patients with high baseline arterial stiffness [30].

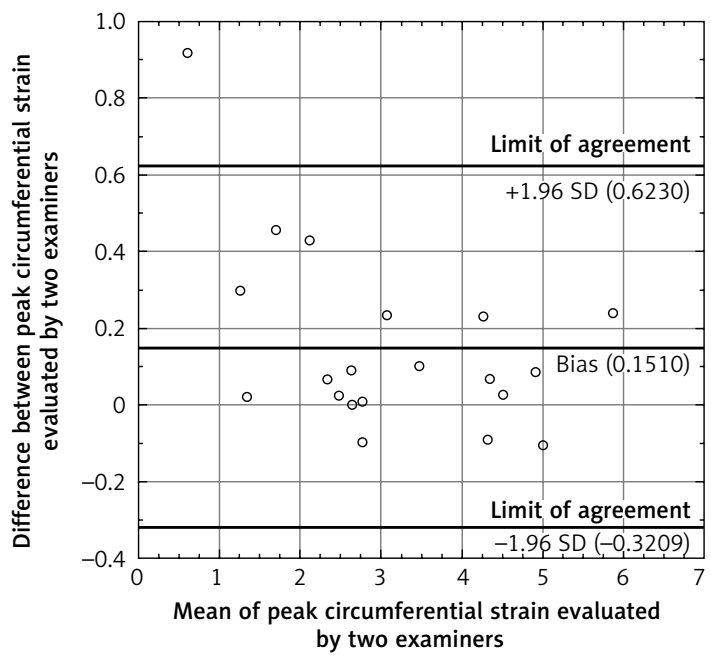

Figure 6. Bland-Altman plot

In contrast, this study shows that 2DST was successful in recognising patients with CS $=0$ among individuals with a mean age of $61 \pm 10.6$ years. Moreover, circumferential strain rate correlates more closely with age than IMT, and circumferential strain was almost as strongly correlated with SBP as IMT. Additionally, 2DST/IMT seems to be superior to methods assessing functional endothelial dysfunction, which are not suitable for the evaluation of patients from their $5^{\text {th }}$ decade [31]. Although circumferential strain was not so closely correlated with age as in younger individuals, the correlation coefficients being -0.38 vs. -0.57 [9] for individuals at mean ages of 61 and 29 respectively, this study suggests that both strain variables and IMT are suitable for evaluation of different aspects of arteriosclerosis in elderly individuals.

The presented technique has several technical advantages over other ultrasonographic methods of arterial wall stiffness assessment. Unlike tissue Doppler imaging and IMT measurements, it is an- 
gle independent and allows for the analysis of all segments of the arterial wall [9]. Moreover, it requires only 3 cardiac cycles, whereas for automated border detection with acoustic quantification, 10 consecutive heart beats are needed [5]. It may greatly facilitate data acquisition in patients with cardiac arrhythmias.

This method also seems to be superior to other non-sonographic techniques evaluating arterial wall compliance. In an examination of the CCA by means of vector velocity imaging, the average peak circumferential strain and circumferential strain rate were negatively correlated with pulsewave velocity (PVW) and with the augmentation index [11]. Although PVW is becoming the gold standard examination in the evaluation of arterial stiffness [6], it is pressure and heart rate dependant, requires special equipment, and in elderly subjects, with a more tortuous abdominal aorta, can be underestimated [32]. The correlation with strain variables suggests that an easy and reliable technique of strain evaluation, like 2DST, may replace PVW in everyday clinical practice. Because of all the aforementioned advantages, 2DST/IMT might be especially useful for repeated examination of patients with type 1 diabetes, in whom combined analysis of arterial wall stiffness and IMT has proved to be valuable in the evaluation of macro- and microangiopathy [28].

In this study, intra- and inter-observer agreement were very good, reaching correlations of $85 \%$ and $94 \%$ respectively. They were comparable with results obtained by Park et al. [22] (93\% and $90 \%$ respectively) and by Yuda et al. [9] (88\% and 93\% respectively). Additionally, this technique allows for assessment of all individuals in the analysed population. In other studies $100 \%$ feasibility was also achieved $[3,13]$. One study demonstrated lower feasibility; however, in this case the acquisition frame rate might have been too low [22]. The Bland-Altman plot shows that this method allows for reliable assessment of the circumferential strain in patients with different levels of CCA stiffness. One outlier was a patient with the stiffest artery, where the small difference in raw data gave a high percentile value.

This study has several limitations, one being that only the left CCA was evaluated. However, earlier studies confirm that no significant differences in averaged circumferential strain exist between the right and left CCA [9]. Moreover, although the feasibility of 2DST was $100 \%$ in this study, this technique would not be suitable for patients with total and subtotal obliteration of the CCA and with atrial fibrillation. Finally, all evaluation and comparison took place using cardiovascular risk surrogates instead of actual cardiovascular events.

In conclusion, increased stiffness and IMT of the CCA were observed in a group of elderly patients with defined cardiovascular risk factors and with calcium deposits in coronary arteries. The measurement of IMT and circumferential strain variables, evaluated with the 2DST technique, allow for a more detailed evaluation of atherosclerosis progression in patients with $\mathrm{CS}=0$. Furthermore, they are based on an ultrasound technique which is quick, non-invasive and relatively cheap. All the above suggest that CCA strain imaging assessed by 2DST and IMT measurement may be suitable for initial evaluation of atherosclerosis severity in patients up to the $6^{\text {th }}$ decade of life.

\section{Conflict of interest}

The authors declare no conflict of interest.

\section{References}

1. O'Rourke MF, Staessen JA, Vlachopoulos C, et al. Clinical applications of arterial stiffness; definitions and reference values. Am J Hypertens 2002; 15: 426-44.

2. Zahnd G, Boussel L, Marion A. Measurement of two-dimensional movement parameters of the carotid artery wall for early detection of arteriosclerosis: a preliminary clinical study. Ultrasound Med Biol 2011; 37: 1421-9.

3. Catalano M, Lamberti-Castronuovo A, Catalano A, et al. Two-dimensional speckle-tracking strain imaging in the assessment of mechanical properties of carotid arteries: feasibility and comparison with conventional markers of subclinical atherosclerosis. Eur J Echocardiogr 2011; 12: 528-35.

4. Gamble G, Zorn J, Sanders G, et al. Estimation of arterial stiffness, compliance, and distensibility from M-mode ultrasound measurements of the common carotid artery. Stroke 1994; 25: 11-6.

5. Luc M, Polonsky T, Lammertin G, Spencer K. Automated border detection for assessing the mechanical properties of the carotid arteries: comparison with carotid intima-media thickness. J Am Soc Echocardiogr 2010; 23: 567-72.

6. Urbina EM, Williams RV, Alpert BS, et al. American Heart Association Atherosclerosis, Hypertension, and Obesity in Youth Committee of the Council on Cardiovascular Disease in the Young. Noninvasive assessment of subclinical atherosclerosis in children and adolescents: recommendations for standard assessment for clinical research: a scientific statement from the American Heart Association. Hypertension 2009; 54: 919-50.

7. Luo J, Li RX, Konofagou EE. Pulse wave imaging of the human carotid artery: an in vivo feasibility study. IEEE Trans Ultrason Ferroelectr Freq Control 2012; 59: 174-81.

8. Kawasaki T, Fukuda S, Shimada K, et al. Direct measurement of wall stiffness for carotid arteries by ultrasound strain imaging. J Am Soc Echocardiogr 2009; 22: 1389-95.

9. Yuda S, Kaneko R, Muranaka A, et al. Quantitative measurement of circumferential carotid arterial strain by two-dimensional speckle tracking imaging in healthy subjects. Echocardiography 2011; 28: 899-906.

10. Kim SA, Park SM, Kim MN, et al. The relationship between mechanical properties of carotid artery and coronary artery disease. Eur Heart J Cardiovasc Imaging 2012; 13: 568-73.

11. Yang WI, Shim CY, Bang WD, et al. Asynchronous arterial systolic expansion as a marker of vascular aging: 
assessment of the carotid artery with velocity vector imaging. J Hypertens 2011; 29: 2404-12.

12. Leitman M, Lysyansky P, Sidenko S, et al. Two-dimensional strain-a novel software for real-time quantitative echocardiographic assessment of myocardial function. J Am Soc Echocardiogr 2004; 17: 1021-9.

13. Bjällmark A, Lind B, Peolsson $M$, et al. Ultrasonograph ic strain imaging is superior to conventional non-invasive measures of vascular stiffness in the detection of age-dependent differences in the mechanical properties of the common carotid artery. Eur J Echocardiogr 2010 11: 630-6.

14. Budoff MJ, Nasir K, McClelland RL, et al. Coronary calcium predicts events better with absolute calcium scores than age-sex-race/ethnicity percentiles: MESA (Multi-Ethnic Study of Atherosclerosis). J Am Coll Cardiol 2009; 53: 345-52.

15. Oliver JJ, Webb DJ. Noninvasive assessment of arterial stiffness and risk of atherosclerotic events. Arterioscler Thromb Vasc Biol 2003; 23: 554-66.

16. Yang EY, Dokainish H, Virani SS, et al. Segmental analysis of carotid arterial strain using speckle-tracking. J Am Soc Echocardiogr 2011; 24: 1276-84.

17. Laurent S. Arterial stiffness: intermediate or surrogate endpoint for cardiovascular events? Eur Heart J 2005; 26: $1152-4$

18. Khamdaeng T, Luo J, Vappou J, et al. Arterial stiffness identification of the human carotid artery using the stress-strain relationship in vivo. Ultrasonics 2012; 52: 402-11.

19. Oishi Y, Mizuguchi Y, Miyoshi H, et al. A novel approach to assess aortic stiffness related to changes in aging using a two-dimensional strain imaging. Echocardiography 2008; 25: 941-5.

20. Claridge MW, Bate GR, Dineley JA, et al. A reproducibility study of a TDI-based method to calculate indices of arterial stiffness. Ultrasound Med Biol 2008; 34: 215-20.

21. Saito $M$, Okayama $H$, Inoue $K$, et al. Carotid arterial circumferential strain by two-dimensional speckle tracking: a novel parameter of arterial elasticity. Hypertens Res 2012; 35: 897-902.

22. Park HE, Cho GY, Kim HK, et al. Validation of circumferential carotid artery strain as a screening tool for sub clinical atherosclerosis. J Atheroscler Thromb 2012; 19 349-56.

23. Rumberger JA, Brundage BH, Rader DJ, Kondos G. Electron beam computed tomographic coronary calcium scanning: a review and guidelines for use in asymptomatic persons. Mayo Clin Proc 1999; 74: 243-52.

24. Kanters SD, Elgersma OE, Banga JD, et al. Reproducibility of measurements of intima-media thickness and distensibility in the common carotid artery. Eur J Vasc Endovasc Surg 1998; 16: 28-35

25. DeLoach SS, Townsend RR. Vascular stiffness: its measurement and significance for epidemiologic and outcome studies. Clin J Am Soc Nephrol 2008; 3: 184-92.

26. Kurnatowska I, Grzelak P, Stefańczyk L, Nowicki M. Tight relations between coronary calcification and atherosclerotic lesions in the carotid artery in chronic dialysis patients. Nephrology (Carlton) 2010; 15: 184-9.

27. Wolski C, Rotkiewicz A, Grzelak P, et al. Comparison of tomographic coronary artery calcification index (calcium score) and ultrasonographic measurement of intima-media complex thickness in diabetic subjects. Pol I Radiol 2011; 76: 15-20.

28. Rogowicz-Frontczak A, Araszkiewicz A, Pilacinski S, et al. Carotid intima-media thickness and arterial stiffness in type 1 diabetic patients with and without microangiopathy. Arch Med Sci 2012; 8: 484-90.

29. Vriz O, Bossone E, Bettio M, et al. Carotid artery stiffness and diastolic function in subjects without known cardiovascular disease. J Am Soc Echocardiogr 2011; 24: 915-21.

30. Dijk JM, Algra A, van der Graaf Y, et al.; SMART study group. Carotid stiffness and the risk of new vascular events in patients with manifest cardiovascular disease. The SMART study. Eur Heart J 2005; 26: 1213-20.

31. Grzelak P, Olszycki M, Majos A, et al. Hand exercise test for the assessment of endothelium-dependent vasodilatation in subjects with type 1 diabetes. Diabetes Technol Ther 2010; 12: 605-11.

32. Oliver JJ, Webb DJ. Noninvasive assessment of arterial stiffness and risk of atherosclerotic events. Arterioscler Thromb Vasc Biol 2003; 23: 554-66. 